\title{
Crystal structure of the Rous sarcoma virus intasome
}

$\underline{\text { Hideki Aihara }}{ }^{1}$, Zhiqi Yin ${ }^{1}$, Ke Shi ${ }^{1}$, Surajit Banerjee ${ }^{2}$, Krishan Pandey $^{3}$, Sibes Bera ${ }^{3}$, Duane P. Grandgenett ${ }^{3}$

${ }^{1}$ University of Minnesota, Minneapolis, MN, USA

${ }^{2}$ NE-CAT, Cornell University, Argonne, IL, USA

${ }^{3}$ St. Louis University, St. Louis, MO, USA

Integration of the reverse-transcribed viral DNA into the host genome is an essential step in the lifecycle of retroviruses, which is catalyzed by the viral integrase protein (IN). IN from HIV-1 and closely related retroviruses share the three-domain organization, consisting of a catalytic core domain flanked by $\mathrm{N}$ - and $\mathrm{C}$-terminal domains essential for the concerted integration of both viral DNA termini. We have determined at $3.8 \AA$ resolution a crystal structure of the threedomain IN from Rous sarcoma virus (RSV) in complex with viral and target DNAs. The structure shows an octameric assembly of IN, in which a pair of IN dimers engage viral DNA ends for catalysis while another pair of non-catalytic IN dimers bridge between the two viral DNA molecules and help capture target DNA. The individual domains of the eight IN molecules play varying roles to hold the complex together, making an extensive network of protein-DNA and protein-protein contacts. Our work and the recent structural studies by other labs collectively highlight remarkable diversity of the intasome assembly among different genera of retroviruses. 